\title{
INFRARED EMISSION AND STAR FORMATION IN THE CENTRAL REGIONS OF THE GALAXY IC 342
}

\author{
E. E. Becklin, ${ }^{1,2,3}$ I. Gatley, ${ }^{2,3}$ K. Matthews, ${ }^{3}$ G. Neugebauer, ${ }^{2,3}$ K. Sellgren, ${ }^{2}$ M. W. Werner ${ }^{2,3}$ \\ AND C. G. WYNN-WILliams ${ }^{1,4}$ \\ Received 1979 July 20; accepted 1979 September 10
}

\begin{abstract}
The face-on Scd galaxy IC 342 has been studied at infrared wavelengths between $1.2 \mu \mathrm{m}$ and $250 \mu \mathrm{m}$ and at centimeter radio wavelengths. At $10 \mu \mathrm{m}$ the nucleus is bright and extended on a scale of $200 \mathrm{pc}$, with a double structure unlike that of the stars seen at $2 \mu \mathrm{m}$. The infrared emission between 8 and $250 \mu \mathrm{m}$ probably arises from heated dust grains in star formation regions in the disk of the galaxy. The radio emission comes from a region with many of the same spatial features as the $10 \mu \mathrm{m}$ source; it appears to include both thermal and nonthermal sources. IC 342 is intermediate in luminosity between the Galaxy and NGC 253; the difference can probably be accounted for by a difference in the current rate of star formation at their centers.
\end{abstract}

Subject headings: galaxies: individual — galaxies: nuclei — galaxies: stellar content infrared: sources - interstellar: matter — stars: formation

\section{INTRODUCTION}

IC 342 is a nearby large spiral galaxy of type Scd. It has received less attention than other galaxies of a similar angular size because it lies close to the galactic plane $\left(b^{\mathrm{II}}=11^{\circ}\right)$ and hence suffers from substantial extinction at visible wavelengths. Its distance is uncertain. Ables (1971), in his photometric study, placed it at a distance of $1.5 \mathrm{Mpc}$, within the Local Group, but newer observations, summarized by Baker et al. (1977), have suggested a larger distance of around $4.5 \mathrm{Mpc}$, the value which will be adopted here.

This paper presents the first infrared observations of IC 342. These include airborne photometry in the $100 \mu \mathrm{m}$ region, and ground-based spectrophotometry and mapping of the galaxy at wavelengths between 1.2 and $20 \mu \mathrm{m}$. Radio continuum observations of the central region are also reported. The galaxy was selected for study at infrared wavelengths mainly because of the strong $\mathrm{CO}$ emission detected from it by Morris and Lo (1978).

\section{OBSERVATIONS}

\section{a) Ground-Based Measurements}

Observations were carried out at the Mount Wilson $1.5 \mathrm{~m}$ and Palomar Mountain Hale $5 \mathrm{~m}$ telescopes. The 1.2-3.4 $\mu \mathrm{m}$ observations employed an $\mathrm{f} / 16$ focal plane chopper system with an InSb detector, while

\footnotetext{
${ }^{1}$ Institute for Astronomy, University of Hawaii.

${ }^{2}$ Downs Laboratory of Physics, California Institute of Technology.

${ }^{3}$ Hale Observatories, California Institute of Technology. The Hale Observatories are operated jointly by California Institute of Technology and Carnegie Institution of Washington. U.K.
}

the 8-20 $\mu \mathrm{m}$ observations used a germanium bolometer and an $\mathrm{f} / 60$ or $\mathrm{f} / 72$ chopping secondary photometer. As noted in the text and tables, various sized diaphragms and signal-reference beam spacings were used. The absolute calibrations and the filters used for the photometric measurements are the same as those listed by Neugebauer et al. (1979). Maps of the source were made by using a 3".8 or 4".2 diaphragm on the $5 \mathrm{~m}$ telescope. Maps at 2.2 and $1.6 \mu \mathrm{m}$ were made by scanning the telescope in declination; the map at $10 \mu \mathrm{m}$ was made by performing integrations spaced by 1.5 in each coordinate. The maps were located by reference to the position of maximum visual brightness of the IC 342 nucleus as seen either directly through an eyepiece for the 1.6 and $2.2 \mu \mathrm{m}$ work or through a television system for the $10 \mu \mathrm{m}$ observations. Spectroscopic observations in the $2.2 \mu \mathrm{m}$ window were made on both telescopes by using a circular variable filter wheel with a resolution of $0.03 \mu \mathrm{m}$ at $2.2 \mu \mathrm{m}$ and an InSb detector.

\section{b) Airborne Infrared Measurements}

Observations were made in the $45-250 \mu \mathrm{m}$ wavelength range using the Kuiper Airborne Observatory. The multichannel photometer and the method of observation are as described in Gatley et al. (1977), with the addition of a channel centered at $200 \mu \mathrm{m}$ $(\Delta \lambda=100 \mu \mathrm{m})$. Photometric measurements of the central region were made at $50 \mu \mathrm{m}, 100 \mu \mathrm{m}$, and 200 $\mu \mathrm{m}$ using a $60^{\prime \prime}$ diaphragm. In a separate observation, which has already been described by Sellgren, Werner, and Gatley (1978), spatial scans of the same region were also made in north-south and east-west directions using a $30^{\prime \prime}$. beam and a broad 45-135 $\mu \mathrm{m}$ bandwidth. The chopper spacing was $2^{\prime}$ for the far-infrared measurements. 


\section{c) Radio Observations}

Maps of the central region of IC 342 were made by using the Cambridge $5 \mathrm{~km}$ telescope at frequencies of 2.7 and $5 \mathrm{GHz}$. At these frequencies the full width at half-maxima of the beams are $3.7 \times 4$ 4.0 and $2.0 \times$ $2 " .2$, respectively, in right ascension and declination. Each map is the result of a single 12 hour observing run with 16 interferometer baselines. The rms noise levels on the full resolution maps are about 1.3 and $2.0 \mathrm{mJy}$ per beam area at 2.7 and $5 \mathrm{GHz}$, corresponding to $17 \mathrm{~K}$ and $26 \mathrm{~K}$, respectively

\section{RESULTS}

\section{a) Mapping of the Source}

Figure 1 shows maps of the central region of IC 342 at $2.2 \mu \mathrm{m}, 10 \mu \mathrm{m}$, and $5 \mathrm{GHz}$. At $2.2 \mu \mathrm{m}$ wavelength the nucleus consists of a single, extended source which lies within 1 " of the peak optical position; a map made at $1.6 \mu \mathrm{m}$ agrees in position and shape with that at $2.2 \mu \mathrm{m}$ within experimental uncertainty. At $10 \mu \mathrm{m}$ two peaks are seen, separated by $4 "(90 \mathrm{pc})$. One peak lies very close to the $2.2 \mu \mathrm{m}$ peak, but with a marginally significant $1^{\prime \prime}$ displacement to the north; the other

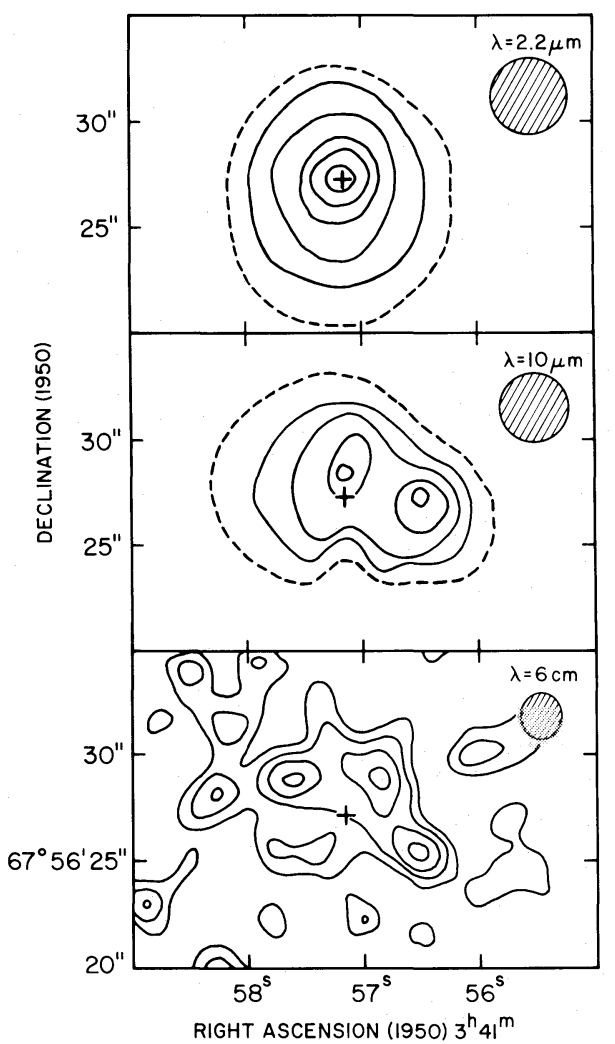

Fig. 1.-Maps of the central region of IC 342 at $2.2 \mu \mathrm{m}$, $10 \mu \mathrm{m}$, and $5 \mathrm{GHz}$. The cross marks the position of the visible peak of the nucleus, and the shaded regions show the halfpower beams. The contour intervals are $14 \mathrm{mJy}$ into a 3 ". beam at $2.2 \mu \mathrm{m}, 0.1 \mathrm{Jy}$ into a 4.2 beam at $10 \mu \mathrm{m}$, and $26 \mathrm{~K}$ at $5 \mathrm{GHz}$. The dashed contour is at half this level. lies to the west. Both regions are extended on a scale of about $5^{\prime \prime}$. The relative position errors between the infrared maps are about $1^{\prime \prime}$. The absolute coordinates used in the infrared maps have been established by adopting the position given for the visible nucleus by van der Kruit (1973) as the reference. Because of the nonstellar appearance of the visible nucleus in the telescope, the infrared coordinates may be in error by up to $3^{\prime \prime}$.

The $5 \mathrm{GHz}$ radio source consists of several peaks of emission spread over an area about $10^{\prime \prime}$ in diameter. The three brightest peaks lie in a region of approximately the same size and shape as the $10 \mu \mathrm{m}$ map, but there are marginally significant emission features outside of this area. Because the $10 \mu \mathrm{m}$ observations did not extend more than a few arcsec outside the outer dashed contour shown in Figure 1, it is not known if there is any $10 \mu \mathrm{m}$ emission from any of the outlying $5 \mathrm{GHz}$ features. The absolute positional accuracy of the radio contours is about $1^{\prime \prime}$.

The $2.7 \mathrm{GHz}$ radio map is shown in Figure 2, together with a version of the $5 \mathrm{GHz}$ map which has been smoothed to the same resolution as the $2.7 \mathrm{GHz}$ map. It may be seen that the general shape of the source is similar at the two frequencies, but that the $6 \mathrm{~cm}$ source is slightly narrower in declination. Variations in spectral index in the source are discussed in $\S \mathrm{IV} b$. The integrated flux densities at the two frequencies are given in Table 3 . The main error is uncertainty in the baselines of the two maps.

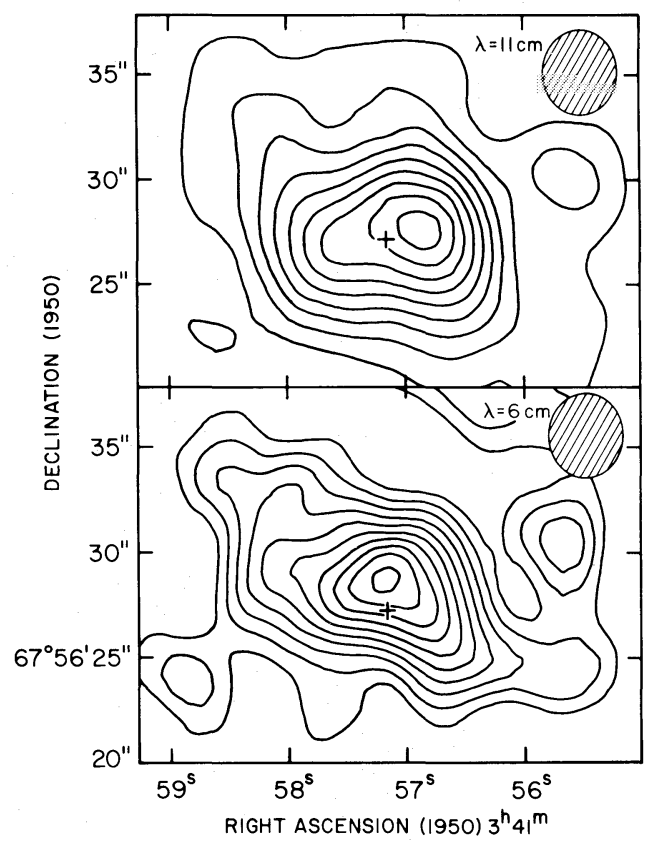

FIG. 2.-Map of the $2.7 \mathrm{GHz}$ radio emission and of the $5 \mathrm{GHz}$ emission convolved to the same resolution $(3.7 \times$ $4^{\prime \prime} 0$ ) as the $2.7 \mathrm{GHz}$ data. The contour intervals are $26 \mathrm{~K}$ at $2.7 \mathrm{GHz}$ and $7 \mathrm{~K}$ at $5 \mathrm{GHz}$, in both cases corresponding to $2 \mathrm{mJy}$ per beam area. 
TABLE 1

1-4 $\mu$ m PHOTOMETRY OF IC 342

$\log S_{v}\left(W \mathrm{~m}^{-2} \mathrm{~Hz}^{-1}\right)$

\begin{tabular}{|c|c|c|c|c|}
\hline \multirow{2}{*}{$\begin{array}{l}\text { DiAPHRAGM } \\
\text { DiaMETER } \\
\left({ }^{\prime \prime}\right)\end{array}$} & \multicolumn{4}{|c|}{ WAVELENGTH $(\mu \mathrm{m})$} \\
\hline & 1.25 & 1.65 & 2.2 & 3.5 \\
\hline 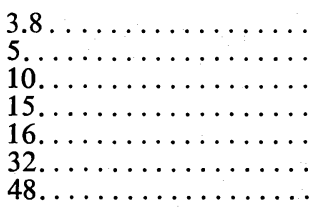 & $\begin{array}{c}-27.31 \pm 0.05 \\
\ldots \\
\ldots \\
-26.76 \pm 0.04 \\
\ldots \\
\ldots\end{array}$ & $\begin{array}{c}-27.13 \pm 0.03 \\
\ldots \\
\cdots \\
-26.65 \pm 0.03 \\
\ldots \\
\ldots\end{array}$ & $\begin{array}{l}-27.15 \pm 0.03 \\
-27.00 \pm 0.03 \\
-26.77 \pm 0.03 \\
-26.68 \pm 0.03 \\
-26.66 \pm 0.03 \\
-26.49 \pm 0.03 \\
-26.41 \pm 0.03\end{array}$ & $\begin{array}{c}-27.38 \pm 0.07 \\
\ldots \\
\ldots \\
\ldots \\
\ldots \\
\ldots\end{array}$ \\
\hline
\end{tabular}

The $3 " 8-15^{\prime \prime}$ measurements were made on the Hale $5 \mathrm{~m}$ telescope using a $15^{\prime \prime} \mathrm{N}-\mathrm{S}$ chopper spacing. The $16^{\prime \prime}-48^{\prime \prime}$ measurements were made on the Mount Wilson $1.5 \mathrm{~m}$ telescope with a $48^{\prime \prime} \mathrm{N}-\mathrm{S}$ chopper spacing. No correction for flux in the reference beam has been made.

\section{b) Photometry}

The photometric observations of IC 342 are listed in Tables 1, 2, and 3. Table 1 shows the flux densities in the wavelength range $1.2-3.5 \mu \mathrm{m}$ for a range of diaphragm sizes, while Table 2 shows the 8-20 $\mu \mathrm{m}$ observations of the two peaks shown in Figure 1 and of an $18^{\prime \prime}$ region encompassing the whole nuclear region. In these tables no correction for flux in the reference beam has been made, and the errors include calibration uncertainties. Table 3 shows the 50, 100, and $200 \mu \mathrm{m}$ flux densities, the $1 \mathrm{~mm}$ limit as published by Elias et al. (1978), and the total $2.7 \mathrm{GHz}$ and $5 \mathrm{GHz}$ flux densities, obtained by integration of the radio maps in Figure 2.

The energy distribution of the central $4^{\prime \prime}$ of the galaxy is shown in Figure 3. The $8-20 \mu \mathrm{m}$ measurements are those of the eastern of the two sources seen in Figure 1, the one coincident with the $2.2 \mu \mathrm{m}$ peak. The energy distribution peaks at about $2 \mu \mathrm{m}$, has a minimum at $3.5 \mu \mathrm{m}$, and then increases rapidly with increasing wavelength. The absorption feature seen in Figure 3 and in Table 2 at $9.5 \mu \mathrm{m}$ is presumably due to silicate material which is seen in many extragalactic objects (Rieke and Low 1975; Kleinmann, Gillett, and Wright 1976; Lebofsky and Rieke 1979).

Between 1.2 and $3.5 \mu \mathrm{m}$ there is little change of infrared colors with diaphragm size. The two sources seen at $10 \mu \mathrm{m}$ have very similar energy distributions both across the silicate band and between 10 and 20 $\mu \mathrm{m}$. Between $2 \mu \mathrm{m}$ and $10 \mu \mathrm{m}$, however, the energy distributions of the two sources are very different, as can be discerned from the difference in the maps of the region at these wavelengths (Fig. 1).

The dependence of $2.2 \mu \mathrm{m}$ flux density with diaphragm diameter is shown in Figure 4 for diaphragms in the range $3.8-48^{\prime \prime}$. The fluxes have been corrected for flux in the reference beam, assuming a power-law dependence for the radial decrease in flux density over the relevant range of signal-reference-beam separations. Since it is the variation in $2.2 \mu \mathrm{m}$ flux density with diaphragm diameter that is relevant in Figure 4, the error bars exclude calibration errors and reflect only the statistical errors and the uncertainties in the correction for reference-beam flux density. Also drawn for comparison is the flux density in the blue photometric band as a function of diameter. This quantity has been calculated from the isophotometric data of Ables (1971). It may be seen that there is some marginal evidence that the $B-[2.2 \mu \mathrm{m}]$ color becomes bluer toward the center of IC 342 .

The sharp increase in emission longward of $4 \mu \mathrm{m}$ in Figure 3 extends to longer wavelengths, as can be deduced from the large flux densities at $50 \mu \mathrm{m}$ and $100 \mu \mathrm{m}$. Cross scans with a $30^{\prime \prime}$ diaphragm at $100 \mu \mathrm{m}$ (Sellgren et al.) indicate that the size of the source at these wavelengths is not significantly greater than $30^{\prime \prime}$ $(700 \mathrm{pc})$. The total emission in the aircraft band,

TABLE 2

8.7-20 $\mu \mathrm{m}$ РHOTOMETRY OF IC 342

$\log S_{v}\left(\mathrm{~W} \mathrm{~m}^{-2} \mathrm{~Hz}^{-1}\right)$

\begin{tabular}{|c|c|c|c|c|c|c|}
\hline \multirow{2}{*}{$\begin{array}{c}\text { SOURCE AND } \\
\text { DIAPHRAGM DIAMETER (") }\end{array}$} & \multicolumn{6}{|c|}{ WAVELENGTH $(\mu \mathrm{m})$} \\
\hline & 8.7 & 9.5 & 11.2 & 12.5 & 10 & 20 \\
\hline $\begin{array}{l}\text { Central Source } 4.2 \ldots \ldots \\
\text { Western Source } 4.2 \ldots \ldots \\
\text { Central Source } 18 \ldots \ldots\end{array}$ & $\begin{array}{c}-26.46 \pm 0.08 \\
-26.38 \pm 0.07 \\
\ldots\end{array}$ & $\begin{array}{c}-26.70 \pm 0.14 \\
-26.56 \pm 0.11 \\
\ldots\end{array}$ & $\begin{array}{c}-26.43 \pm 0.08 \\
-26.27 \pm 0.07 \\
\ldots\end{array}$ & $\begin{array}{c}-26.31 \pm 0.08 \\
-26.21 \pm 0.07 \\
\ldots\end{array}$ & $\begin{array}{l}-26.36 \pm 0.09 \\
-25.79 \pm 0.08\end{array}$ & $\begin{array}{l}-25.95 \pm 0.17 \\
-25.76 \pm 0.14 \\
-25.21 \pm 0.10\end{array}$ \\
\hline
\end{tabular}

The 4". 2 measurements were made on the Hale $5 \mathrm{~m}$ telescope using a $14^{\prime \prime} \mathrm{N}-\mathrm{S}$ chopper spacing.

The $18^{\prime \prime}$ measurements were made on the Mount Wilson $1.5 \mathrm{~m}$ telescope with an $18^{\prime \prime}$ chopper spacing. 
TABLE 3

Long-Wavelength Photometry of IC 342

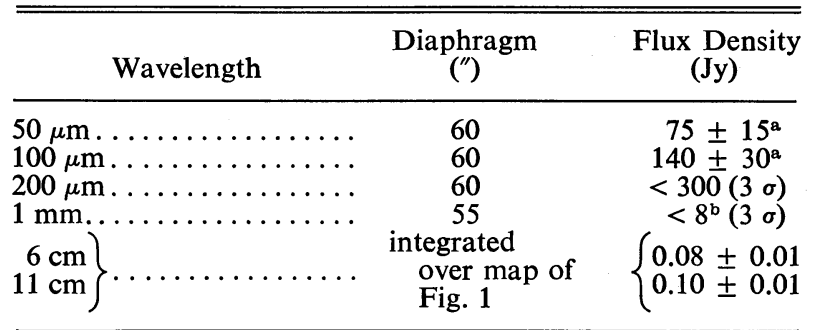

a The quoted errors are primarily due to calibration; the statistical uncertainty was less than $10 \%$.

${ }^{\text {b }}$ From Elias et al. (1978).

$45-135 \mu \mathrm{m}$, is $4.5 \times 10^{-12} \mathrm{~W} \mathrm{~m}^{-2}$, which corresponds to a bolometric luminosity of $2.7 \times 10^{9} L_{\odot}$ for an assumed distance of $4.5 \mathrm{Mpc}$. Inclusion of the estimated infrared emission outside this band gives a total luminosity of $4 \times 10^{9} L_{\odot}$ between 3 and $1000 \mu \mathrm{m}$ for the central $700 \mathrm{pc}$ of the galaxy. The color temperature between 50 and $100 \mu \mathrm{m}$ is $55 \mathrm{~K}$.

\section{c) Spectroscopy}

Figure 5 shows the continuously variable filter spectrum of IC 342 from 2.1 to $2.4 \mu \mathrm{m}$ as measured with a $16^{\prime \prime}$ diaphragm on the Mount Wilson $1.5 \mathrm{~m}$ telescope. The continuous line is a Rayleigh-Jeans $\left(\lambda^{-4}\right)$ curve through the points at $\lambda<2.3 \mu \mathrm{m}$. The two short spectra in the vicinity of $2.16 \mu \mathrm{m}$ show the region near the $\mathrm{B} \gamma$ line as measured on the central and western $10 \mu \mathrm{m}$ peaks using $5^{\prime \prime}$ diaphragms on the $5 \mathrm{~m}$ telescope. No B $\gamma$ line was detected to a $3 \sigma$ limit

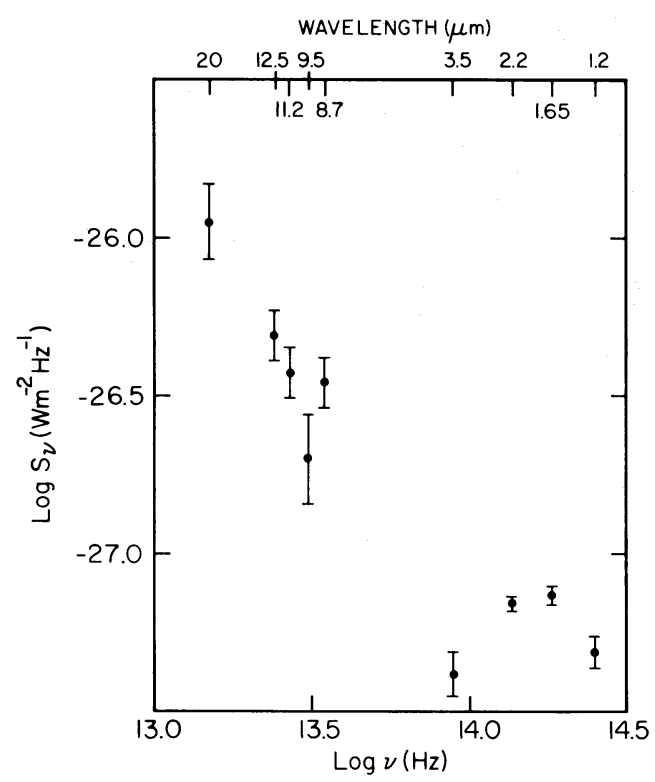

FIG. 3.-Energy distribution from 1.2 to $20 \mu \mathrm{m}$ of the central region of IC 342 . The diaphragm used was 3 ".8 for $\lambda<5 \mu \mathrm{m}$ and 4.2 for $\lambda>5 \mu \mathrm{m}$.

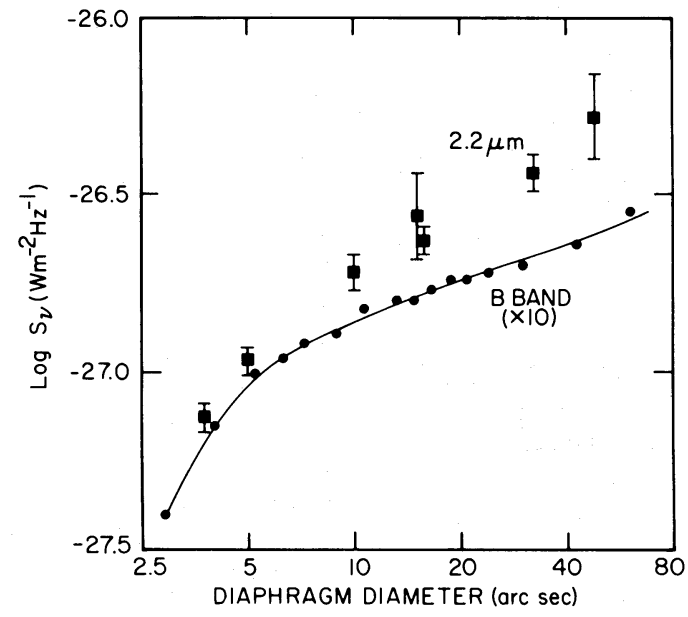

Fig. 4.-The dependence of $2.2 \mu \mathrm{m}$ flux density on diaphragm diameter, corrected for flux in the reference beam as described in the text. The $B$ band data are taken from Ables (1971).

of $1.5 \times 10^{-16} \mathrm{~W} \mathrm{~m}^{-2}$ for the $5^{\prime \prime}$ measurements and $5 \times 10^{-16} \mathrm{~W} \mathrm{~m}^{-2}$ for the $16^{\prime \prime}$ measurement. A clear absorption feature is seen at wavelengths longward of $2.3 \mu \mathrm{m}$. This feature is attributable to $\mathrm{CO}$ molecules in the photospheres of late-type giant and supergiant stars.

\section{DISCUSSION}

\section{a) Starlight and Extinction}

The spectrum in Figure 5 clearly shows a depression at $2.3 \mu \mathrm{m}$ which is identified as a $\mathrm{CO}$ absorption band arising in the photospheres of late-type giant and

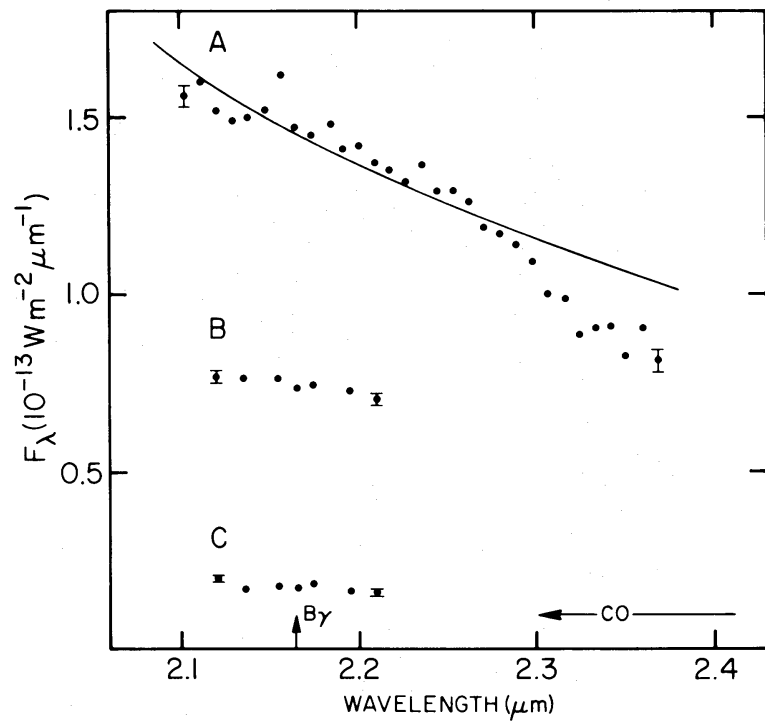

FIG. 5-2.1-2.4 $\mu \mathrm{m}$ spectra of IC 342. The error bars shown are typical of all the points on the appropriate spectrum. Curve A refers to a $16^{\prime \prime}$ diaphragm centered on the $2 \mu \mathrm{m}$ peak; curves $\mathrm{B}$ and $\mathrm{C}$ refer to 3 ".8 diaphragms centered on the central and the western $10 \mu \mathrm{m}$ peaks, respectively. 
supergiant stars. The CO index, as defined by Frogel et al. (1975), is about $0.2 \mathrm{mag}$, making it similar in strength to that in many other spiral galaxies (Aaronson 1978). The implication of this result is that a substantial fraction of the $2.2 \mu \mathrm{m}$ emission from the center of IC 342 comes from late-type giant or supergiant stars. A similar conclusion was reached for the other infrared-emitting galaxies NGC 6946 (Lebofsky and Rieke 1979) and NGC 253 (Wynn-Williams et al. 1979), both of which are galaxies which show farinfrared emission (Telesco and Harper 1980).

In his photometric survey Ables (1971) measured the visual magnitude of the central $34^{\prime \prime}$ of IC 342 to

- be 12.4 , and estimated the extinction in the blue photometric band toward this region to be $A_{B}=2.2$ mag. For an object at this low galactic latitude, all this extinction could arise within the Milky Way. This extinction, together with the interstellar extinction curve no. 15 of van der Hulst (Johnson 1968) and the $2.2 \mu \mathrm{m}$ magnitude estimated from the curve in Figure 3 , leads to an intrinsic $V-[2.2 \mu \mathrm{m}]$ color of $2.9 \pm$ $0.3 \mathrm{mag}$ for the central $34^{\prime \prime}$ region of IC 342 . This value is in excellent agreement with the value of $2.8-2.9 \mathrm{mag}$ given by Aaronson (1978) for the mean $V-[2.2 \mu \mathrm{m}]$ color of Scd galaxies. The 1.2, 1.6, and $2.2 \mu \mathrm{m}$ colors of IC 342 are also in close agreement with those in the sample galaxies studied by Aaronson when the appropriate reddening correction is applied. Thus it can be concluded that the intrinsic infrared colors of IC 342 are normal in the wavelength range $0.4-2.4 \mu \mathrm{m}$.

The presence of the feature normally identified as silicate absorption in the two $10 \mu \mathrm{m}$ sources indicates that the extinction to the sources of radiation at that wavelength is much greater than that toward the stars. The use of a ratio of $A_{v} / \tau_{10 \mu \mathrm{m}}$ of 15 (Rieke 1974; Gillett et al. 1975; Becklin et al. 1978) and of the photometric data in Figure 2 and Table 2 leads to an estimate of $15 \mathrm{mag}$ for the equivalent visual extinction to the $10 \mu \mathrm{m}$ emitting regions.

\section{b) Radio Emission}

One of the most interesting features of Figure 1 is that the maps at both $10 \mu \mathrm{m}$ and $5 \mathrm{GHz}$ are of comparable extent, whereas that at $2.2 \mu \mathrm{m}$ is relatively centrally located. This situation is similar to that in M82 (Rieke and Lebofsky 1979) in that both the radio and $10 \mu \mathrm{m}$ maps show emission over an extended region which is quite different from the condensed $2.2 \mu \mathrm{m}$ emission.

Comparison of the 2.7 and $5 \mathrm{GHz}$ flux densities in Table 3 indicates that the central region of IC 342 has a nonthermal spectral index of about $0.3 \pm 0.2$ for the whole region shown in Figure 1. Certain parts of the source, notably the central peak and the extension west of R.A. $03^{\mathrm{h}} 41^{\mathrm{m}} 56^{\mathrm{s}}$, however, appear to have a significantly flatter spectral index than this, close to the value of zero expected for bremsstrahlung emission. The most likely explanation for this variation is that the central few hundred parsecs of IC 342 , like the corresponding region of the Galaxy, contains a mixture of thermal and nonthermal sources in comparable proportions at $5 \mathrm{GHz}$, namely about $40 \mathrm{mJy}$ each. A similar conclusion, based on lowresolution observations of the spectral index variations over the disk of IC 342, was drawn by Baker et al. (1977).

The limit on the $\mathrm{B} \gamma$ flux density from the $16^{\prime \prime}$ region corresponds to an upper limit of $60 \mathrm{mJy}$ for the thermal emission at $5 \mathrm{GHz}$, assuming a temperature of $10^{4} \mathrm{~K}$ and no reddening. This limit will be raised if the ionized gas lies behind substantial obscuration but, in any case, is compatible with the hypothesis that the thermal flux for the central region is of order $40 \mathrm{mJy}$.

\section{c) Origin of the Infrared Luminosity in IC 342}

As discussed in $\S$ III $b$, the estimated total bolometric luminosity from the central 700 pc region of IC 342 is $4 \times 10^{9} L_{\odot}$, while the $3-20 \mu \mathrm{m}$ luminosity of the central $400 \mathrm{pc}$ region is $0.5 \times 10^{9} L_{\odot}$, approximately equally divided between the eastern and western sources. For reasons discussed elsewhere in the context of other galaxies (e.g., Rieke and Lebofsky 1979) the process which produces the infrared radiation is almost certainly emission from warm dust grains. What is less certain is the source of luminosity which heats the dust. The asymmetry and size of the $10 \mu \mathrm{m}$ emission argues strongly against heating predominantly either by a compact central source or by the general background of late-type stars as seen at $2.2 \mu \mathrm{m}$. In addition, the luminosity available from the optically identifiable stellar background is probably insufficient to provide the total infrared luminosity; if the general population of stars in IC 342 is similar to that in other Sc galaxies, the models of Turnrose (1976) may be used along with the measured $2.2 \mu \mathrm{m}$ flux density to estimate the total luminosity available as starlight (Wynn-Williams et al. 1979). The bolometric luminosity of the stellar population seen at visual wavelengths and at $2.2 \mu \mathrm{m}$ within a $48^{\prime \prime}$ diameter area is found in this way to be $10^{9} L_{\odot}$. This luminosity fails by a factor of 4 to account for the total far-infrared radiation. This argument is independent of assumptions about the distance to the source.

Another potential distributed source of luminosity is bright regions of current star formation. Such a possibility is hinted at by the slight blue excess of the central region (Fig. 4), by the strong $10 \mu \mathrm{m}$ emission which is reminiscent of that from $H$ II region/ molecular cloud complexes in our Galaxy, and by the probable presence of free-free radio emission. If the free-free flux density is $40 \mathrm{mJy}$, the total ionization rate is $6 \times 10^{52} \mathrm{~s}^{-1}$ (Rubin 1968); the use of Jennings's (1975) empirical relationship between the total ionization rate and the bolometric luminosity of $\mathrm{H}$ II region/molecular cloud complexes in the Galaxy leads to an estimate of $8 \times 10^{9} L_{\odot}$ for the luminosity available for star formation regions in the center of IC 342. This number is subject to large uncertainties, notably in the separation of the thermal from the nonthermal radio emission and in the wide dispersion in Jennings's data. Nevertheless, it appears that 
heating by molecular cloud/H II region complexes is sufficient to provide some or all of the $4 \times 10^{9} L_{\odot}$ of far-infrared emission from the center of IC 342 . Again, this argument is not dependent on knowledge of the distance to IC 342 .

\section{d) Distribution of Dust in IC 342}

In this section we discuss the spatial distribution of the dust grains which give rise to both the infrared emission and the $10 \mu \mathrm{m}$ absorption feature. From the 50-100 $\mu \mathrm{m}$ color, and with the assumption that the grain emissivity varies as (wavelength) ${ }^{-1}$ over this wavelength range, a temperature of $42 \pm 3 \mathrm{~K}$ may be estimated for the grains which emit at far-infrared wavelengths. If the size of the emitting region at $50 \mu \mathrm{m}$ is $30^{\prime \prime}$ the effective optical depth of the dust at $50 \mu \mathrm{m}$ is 0.01 , as averaged over the $30^{\prime \prime}$ region. The optical depth of each individual warm cloud will be proportionately larger if the filling factor is small.

By using the relationship $A_{v}=300 \tau_{50 \mu \mathrm{m}}$ (Gatley et al. 1979), it may be concluded that the visual extinction which would arise within the far-infrared emitting region is at least $3 \mathrm{mag}$. This value is greater than the visual extinction as estimated to the stars seen at $2.2 \mu \mathrm{m}$, but is not inconsistent with that estimated on the basis of the silicate feature in front of the $10 \mu \mathrm{m}$ emitting region. A conclusion that may be drawn from this result is that the warm dust does not significantly redden the visible starlight. To explain the small reddening at wavelengths shorter than $2 \mu \mathrm{m}$ we require either that the clouds be opaque at these wavelengths, or that they have a small filling factor, or both. The result is also consistent with the idea that the dust causing the silicate absorption feature is the same as the dust giving rise to the $50 \mu \mathrm{m}$ emission, since the hot dust seen at $10 \mu \mathrm{m}$ most probably lies within the cooler $50 \mu \mathrm{m}$ emitting regions.

An attractive model for the dust distribution in the central regions of IC 342 is that the region is populated with discrete, internally heated molecular clouds comparable in nature to those found in the Galaxy. Such clouds are most likely to exist in a disk in the plane of IC 342 with the stars of the central bulge lying both in front of and behind this disk.

The model discussed here is quite similar to that proposed by Morris and Lo (1978) to account for their CO emission observations of IC 342, and it is very probably the same clouds that are being detected at both infrared and millimeter wavelengths. The contours of $10 \mu \mathrm{m}$ emission (Fig. 1) presumably show the spatial distribution of the hot cores of these clouds, which are probably constituted of compact $\mathrm{H}$ II regions and protostars. The double nature of the 10 $\mu \mathrm{m}$ source is not specifically explained by our model but is assumed to be an accident of the distribution of the clouds in space. It is an interesting coincidence that a far-infrared view of the central parts of the Galaxy seen face-on may resemble Figure 1 quite closely, with Sgr A at the position of maximum stellar density and Sgr B2 about 100 pc away from the nucleus.

\section{e) Comparison with Other Galaxies}

Telesco and Harper (1980) have measured the 1-300 $\mu \mathrm{m}$ bolometric luminosities of seven galaxies, ranging from $5 \times 10^{9} L_{\odot}$ for M51, through $30 \times 10^{9}$ $L_{\odot}$ for M82 and NGC 253 , to $300 \times 10^{9} L_{\odot}$ for the Seyfert galaxy NGC 1068. The bolometric luminosity of IC $342,4 \times 10^{9} L_{\odot}$, is comparable to the faintest of Telesco and Harper's galaxies, but it is still a factor of 4 greater than the luminosity from the central 700 pc of the Galaxy, as derived from the data of Low et al. (1977) and Maihara, Oda, and Okuda (1979). This conclusion is strongly dependent on the assumed distance to IC 342 , however.

A useful distance-independent comparison of IC 342 with the Galaxy can be made by calculating the ratio of $100 \mu \mathrm{m}-2.2 \mu \mathrm{m}$ flux densities from the central $700 \mathrm{pc}$ region of each. This ratio is an indicator of the relative luminosity being emitted from hot dust and from the general background of stars; a ratio of 100 corresponds to approximately equal contributions from each component. Using the reddening-corrected $2.4 \mu \mathrm{m}$ data for our Galaxy of Oda et al. (1979) together with the far-infrared data mentioned above, $S_{100} / S_{2.2}=50$ for the Galaxy. By contrast, the results presented in this paper show that $S_{100} / S_{2.2}=400$ for IC 342. The difference in this ratio suggests that in IC 342 heating of the dust by the general background of stars plays a less significant role than it does in the Galaxy, where its contribution is comparable to that from early-type stars (Gatley et al. 1977). This comparison therefore supports the conclusion of $\S \mathrm{IV} c$ that the stellar population seen at visible and near-infrared wavelengths is not luminous enough to be the main power source in IC 342.

From the discussion above there is some evidence that much of the difference between the infrared luminosities of the galaxies can be attributed to the quantity of star formation in the nucleus. In the Galaxy, for example, the heating of the dust in the central few hundred parsecs by late-type stars is significant. In IC 342 there is strong evidence that another, extended source of heating is dominant and some indirect evidence that this source could be young stars. In M82 and NGC 253 the strong Brackett-line emission (Willner et al. 1977; WynnWilliams et al. 1979) and CO emission (Rickard et al. 1977) indicate that there has been a very strong burst of star formation recently. What causes such an outburst and what controls its size is still quite uncertain.

\section{CONCLUSIONS}

1. The infrared luminosity of the central region of IC 342 is $4 \times 10^{9} L_{\odot}$; this is intermediate between that of the Galaxy and those of M82 and NGC 253. Most of the infrared luminosity of IC 342 is emitted longward of $30 \mu \mathrm{m}$.

2. The colors of IC 342 between $2.2 \mu \mathrm{m}$ and the $B$ band are the same as those of other galaxies of similar morphological type. Photospheric emission from 
late-type giant stars dominates at $2.2 \mu \mathrm{m}$. The emission longward of $3.5 \mu \mathrm{m}$ cannot be primarily starlight.

3. The strong $10 \mu \mathrm{m}$ emission is extended on a scale of $200 \mathrm{pc}$, with the brightest point displaced from the peak of the stellar distribution as seen at $2 \mu \mathrm{m}$. However, the $10 \mu \mathrm{m}$ emission spatially resembles the $5 \mathrm{GHz}$ continuum emission rather than the $2 \mu \mathrm{m}$ emission; the radio continuum appears to be a mixture of thermal and nonthermal sources.

4. The far-infrared luminosity cannot be accounted for entirely by the stars seen at visible and nearinfrared wavelengths. The extra luminosity probably arises in molecular cloud/H II region complexes as a result of a burst of star formation. These molecular clouds may also produce the $\mathrm{CO}$ emission from IC 342.

We thank H. Ables for lending us a plate of IC 342, S. Beckwith and our night assistants, G. Tuton, J. Carrasco, and J. Frazier, for help with the groundbased observations, A. Harper, R. Loewenstein, and the members of the Kuiper Airborne Observatory for help with the aircraft observations, J. Shakeshaft for help with the radio observations, and $M$. Morris for bringing this object to our attention. This work was supported by NASA grants NGR 05-005-281, NGL 05-002-207, NASW 3159, and NSF grants AST 77-20516 and AST 7826028.

\section{REFERENCES}

Aaronson, M. 1978, Ph.D. thesis, Harvard University.

Ables, H. D. 1971, Pub. US Naval Obs., Series II, $20,79$.

Baker, J. R., Haslam, C. G. T., Jones, B. B., and Wielebinski, R. 1977, Astr. Ap., 59, 261.

Becklin, E. E., Matthews, K., Neugebauer, G., and Willner, S. P. 1978, Ap. J., 220, 831 .

Elias, J. H. et al. 1978, Ap. J., 220, 25.

Frogel, J. A., Persson, S. E., Aaronson, M., Becklin, E. E., Matthews, K., and Neugebauer, G. 1975, Ap. J. (Letters), 195, L15.

Gatley, I., Becklin, E. E., Werner, M. W., and Wynn-Williams, C. G. 1977, Ap. J., 216, 277.

Gatley, I., Becklin, E. E., Sellgren, K., and Werner, M. W. 1979, Ap. J., 233, 575

Gillett, F. C., Forrest, W. J., Merrill, K. M., Capps, R. W., and Soifer, B. T. 1975, Ap. J., 200, 609.

Jennings, R. E. 1975, in H II and Related Topics, Lecture Notes in Physics, Vol. 42, ed. T. L. Wilson and D. Downes (Berlin: Springer-Verlag), p. 137.

Johnson, H. L. 1968, Stars and Stellar Systems, Vol. VII, ed. B. M. Middlehurst and L. H. Aller (Chicago: University of Chicago Press), p. 167.

Kleinmann, D. E., Gillett, F. C., and Wright, E. L. 1976, Ap. J., 208, 42 .

Lebofsky, M. J., and Rieke, G. H. 1979, Ap. J., 229, 111.
Low, F. J., Kurtz, R. F., Poteet, W. M., and Nishimura, T. 1977, Ap. J. (Letters), 214, L115.

Maihara, T., Oda, N., and Okuda, H. 1979, Ap. J. (Letters), 227, L129.

Morris, M., and Lo, K. Y. 1978, Ap. J., 223, 803

Neugebauer, G., Oke, B. J., Becklin, E. E., and Matthews, K. 1979, Ap. J., 230, 79.

Oda, N., Maihara, T., Sugiyama, T., Okuda, H. 1979, Astr. Ap., 72, 309.

'Rickard, L. J., Palmer, P., Morris, M., Turner, B. E., and Zuckerman, B. 1977, $A p . J ., 213,673$.

Rieke, G. H. 1974, Ap. J. (Letters), 193, L81.

Rieke, G. H., and Lebofsky, M. J. 1979, Ann. Rev. Astr. Ap., 17, p. 477.

Rieke, G. H., and Low, F. J. 1975, Ap. J., 197, 17

Rubin, R. H. 1968, Ap. J., 154, 391.

Sellgren, K., Werner, M. W., and Gatley, I. 1978, Bull. $A A S$, 10,422 .

Telesco, C. M., and Harper, D. A. 1980, Ap. J., 235, 292.

Turnrose, B. E. 1976, Ap. J., 210, 33.

Van der Kruit, P. C. 1973, Astr. Ap., 29, 249.

Willner, S. P., Soifer, B. T., Russell, R. W., Joyce, R. R. and Gillett, F. C. 1977, Ap. J. (Letters), 217, L121.

Wynn-Williams, C. G., Becklin, E. E., Matthews, K., and Neugebauer, G. 1979, M.N.R.A.S., 189, 163.

E. E. BeCKLIN and C. G. WynN-Williams: Institute for Astronomy, University of Hawaii, 2680 Woodlawn Drive, Honolulu, HI 96822

I. GatLey: United Kingdom Infrared Telescope Project, 900 Leilani Street, Hilo, HI 96720

K. Matthews, G. Neugebauer, and K. Sellgren: California Institute of Tecnology, Downs Laboratory 320-47, Pasadena, CA 91125

M. W. Werner: 245-6 NASA Ames Research Center, Moffett Field, CA 94035 